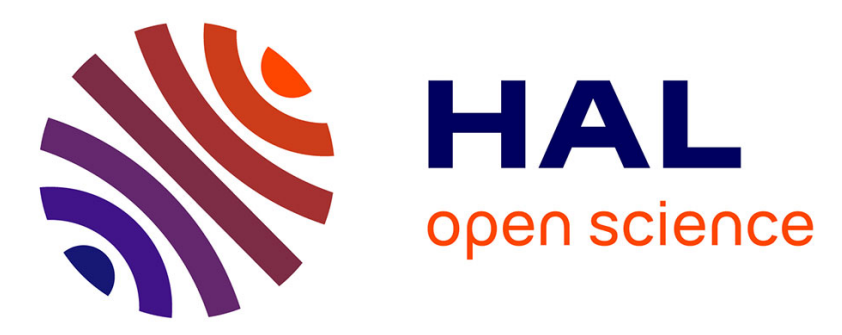

\title{
Acquiring Human-Robot Interaction skills with Transfer Learning Techniques
}

\author{
Omar Mohammed, Gérard Bailly, Damien Pellier
}

\section{To cite this version:}

Omar Mohammed, Gérard Bailly, Damien Pellier. Acquiring Human-Robot Interaction skills with Transfer Learning Techniques. ACM/IEEE International Conference on Human-Robot Interaction, Mar 2016, Vienne, Austria. pp.359 - 360, 10.1145/3029798.3034823 . hal-01490211

\section{HAL Id: hal-01490211 https://hal.science/hal-01490211}

Submitted on 17 Mar 2017

HAL is a multi-disciplinary open access archive for the deposit and dissemination of scientific research documents, whether they are published or not. The documents may come from teaching and research institutions in France or abroad, or from public or private research centers.
L'archive ouverte pluridisciplinaire HAL, est destinée au dépôt et à la diffusion de documents scientifiques de niveau recherche, publiés ou non, émanant des établissements d'enseignement et de recherche français ou étrangers, des laboratoires publics ou privés. 


\title{
Acquiring Human-Robot Interaction skills with Transfer Learning Techniques
}

\author{
Omar Mohammed, Gérard Bailly, and Damien Pellier \\ University of Grenoble Alpes, Grenoble, France \\ \{omar-samir.mohammed, gerard.bailly\}@gipsa-lab.fr, damien.pellier@imag.fr
}

\section{INTRODUCTION}

Recently, an increasing interest in the research community is how to enable robots to interact and communicate with humans, which is a major step towards integrating robots in our daily life. Within the large domain of HumanRobot Interaction (HRI), this research area is called Social robotics [Kanda and Ishiguro 2012]. Communicating with humans is a complex process, involving several modalities such as speech, facial expressions, gaze, head movements, hand gestures, etc. Social robotics focuses on modeling how humans use these communication channels in order to smoothly interact with each other, and how to endow a robot with similar skills. Modeling such complex multimodal interactions has proven to be a challenge.

Machine learning is invading this domain. Recent research performed on interactive data [Mihoub et al. 2016] (i.e. challenging the problem of generating co-verbal gestures of one participant given the co-verbal gestures of the other and verbal activities of both participants) showed a great potential for machine learning in learning complex HRI behaviors. However, the behavioural models are task- and situationspecific: if anything changes in the learning problem (the input distribution or shape, the interaction scenario, etc), we need to learn everything again from scratch. This means that policies learnt in one environment might not work or work sub-optimally - in other environments and conditions [Shimodaira 2000].

A growing interest of research in machine learning is on how to transfer knowledge learned from one task (source task) to a new task (target task) [Weiss et al. 2016]. The idea is to capture the similarities between the source and the target tasks, and exploit them in order to learn the target task faster. The shared knowledge can be implicit, like finding latent variables for the input distributions of both tasks [Pan et al. 2011]. It can also be explicit, like representing robotic knowledge as skills [Konidaris and Barreto 2009].

Our challenge is thus to develop methods to transfer the knowledge model the robot learns on one interactive situa-

ACM ISBN 978-1-4503-2138-9.

DOI: $10.1145 / 1235$ tion with humans to new tasks/situations - notably to new interactive tasks -, in order to enable rapid learning and adaptation to these new tasks.

\section{STATE OF THE ART}

A recent survey of transfer learning can be found in [Weiss et al. 2016]. Most of the techniques outlined in this review paper focus on adapting either the input marginal distribution, output conditional distribution or both, from the new task to the source one. This approach implicitly supposes that a large part of the hidden/latent representations that contribute to map input cues to output cues remain the same whatever the task. This is surely the case of phones in speech recognition or local appearance models in object/agent recognition within images or videos. But what about mapping between more complex sensorimotor sequences such as involved in HRI?

Another approach to transfer knowledge and share internal representation exploits the concept of skills [Sutton et al. 1999, Konidaris and Barreto 2009]. The basic idea is to decompose a task into a set of elementary sub-tasks (i.e. skills), learn how to solve these smaller tasks, and thus solve the task by assembling skills in the same way as assembling words to build a sentence. The introduction of such a syntactic organization of skills has shown to reduce the learning time [Konidaris et al. 2011]. Facing a new task, the challenge is to prime the decomposition using pre-stored skills, identify and learn new skills (similarly to out-of-vocabulary words in speech recognition [Réveil et al. 2014]), and recompile the grammar in order to incorporate old and new skills into the task's model [Konidaris and Barto 2007].

\section{RESEARCH PLAN}

To the best of our knowledge, the current state of the art handles only single modality skills (skills that deals with one type of sensory input), but nothing deals with multimodal skills (where we have different types of sensory inputs, which is the case in HRI), or how to extract skills from HRI situations.

\subsection{Learning and discovering skills}

The challenge of my $\mathrm{PhD}$ thesis is introduce the concept of skills in HRI. The questions we want to answer are: How to decompose the interactive multimodal task into elementary multimodal skills, while incorporating a prior knowledge when available? How to represent such multimodal skills? How to chain those skills in order to solve the source task? How to adapt those skills from this task to a new 
one? How to identify missing skills and learn these new skills? How to cope with different perception/action capabilities from one robot to another?

We are currently testing our ideas on the OPPORTUNITY dataset [Chavarriaga et al. 2013] for human activity recognition. It is constructed from different wearable, object and ambient sensors. The recordings are for 4 users doing daily activities. These activities are: low-level activities relating 13 actions to 23 objects, 17 mid-level gesture classes, and 5 high-level activity classes. Although this dataset does not involve HRI, it has some important characteristics that fit our needs: input is multimodal, it is performed by humans and chains elementary skills such as open/close containers (door, fridge, drawer) and act on objects (switch, cup). The objective is to test our approach: 1) test different techniques to automatically segment human activities into elementary skills, 2) to recognize missing skills once they happen - notably using goodness of fit (GOF) of data regarding the source model - and 3) to use the previous knowledge we have about source skills in order to recognize and train new skills.

\subsection{Generating actions}

Human activity recognition is just an intermediate step for informing perception-for-action with scene understanding. Our target challenge is to generate multimodal behaviour of one target agent given the observed multimodal behaviour of his/her/its communication partners as the interaction unfolds. To this end, we will explore different regression techniques to map lower body movements to upper body movements in the OPPORTUNITY dataset, given that both correlate within skills. We are currently exploring multitasking techniques [Li et al. 2014] with deep learning in order to jointly perform classification (segmenting skills) and regression (mapping perception to action).

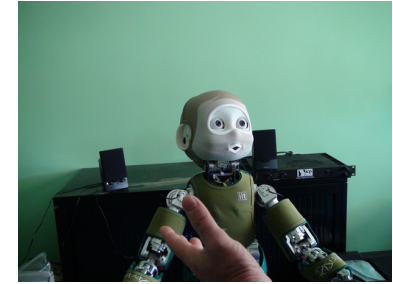

(a) The iCub robot Nina

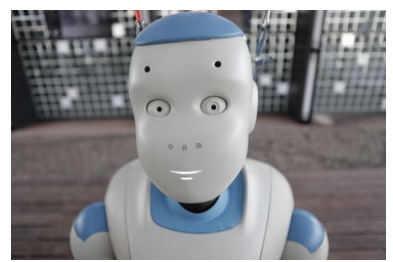

(b) The Romeo robot
Figure 1: The social robots used in the study.

\subsection{HRI skills}

If the this approach is successful, we are planning to move on to test our developed algorithms on actual HRI scenarios. Transfer learning algorithms will be confronted to real human interaction data, notably face-to-face interviews ${ }^{1}$ and board games [Stefanov and Beskow 2016].

Finally, we plan to confronted to realistic HRI data, collected via immersive teleoperation of social robots ${ }^{2}$. This robot-mediated interaction [Bailly et al. 2015] solves the usual HHI-to-HRI scaling issues. This teleoperation strategy - where the human pilot provides social skills to a humanoid robot - indeed aims at providing trustful target HRI

${ }^{1}$ See http://www.gipsa-lab.fr/projet/SOMBRERO/data.html ${ }^{2}$ See http://www.gipsa-lab.fr/projet/SOMBRERO data. Note that two humanoid robots are considered in this project, namely the iCub robot Nina (see Fig. 1.a) and the Romeo robot from Softbank robotics (see Fig. 1.b).

\subsection{Evaluation}

In the framework of the pioneer workshop, we propose to discuss this research plan and welcome comments on some key issues, notably on an evaluation paradigm for interactions that goes beyond objective assessment (see notably [Nguyen et al. 2016]).

\section{REFERENCES}

[Bailly et al. 2015] Gérard Bailly, Frédéric Elisei, and Miquel Sauze. 2015. Beaming the Gaze of a Humanoid Robot. In Proceedings of the Tenth Annual ACM/IEEE International Conference on Human-Robot Interaction Extended Abstracts. ACM, 47-48.

[Chavarriaga et al. 2013] Ricardo Chavarriaga, Hesam Sagha, Alberto Calatroni, Sundara Tejaswi Digumarti, Gerhard TrẪuster, Jos ̃̂l' del R. MillÃąn, and Daniel Roggen. 2013. The Opportunity challenge: A benchmark database for on-body sensor-based activity recognition. Pattern Recognition Letters 34, 15 (2013), $2033-2042$. DOI : http://dx.doi.org/10.1016/j.patrec.2012.12.014 Smart Approaches for Human Action Recognition.

[Kanda and Ishiguro 2012] Takayuki Kanda and Hiroshi Ishiguro. 2012. Human-robot interaction in social robotics. CRC Press.

[Konidaris and Barreto 2009] George Konidaris and Andre S Barreto. 2009. Skill discovery in continuous reinforcement learning domains using skill chaining. In Advances in Neural Information Processing Systems. 1015-1023.

[Konidaris and Barto 2007] George Konidaris and Andrew G Barto. 2007. Building Portable Options: Skill Transfer in Reinforcement Learning.. In IJCAI, Vol. 7. 895-900.

[Konidaris et al. 2011] George Konidaris, Scott Kuindersma, Roderic Grupen, and Andrew Barto. 2011. Robot learning from demonstration by constructing skill trees. The International Journal of Robotics Research (2011), 0278364911428653.

[Li et al. 2014] Sijin Li, Zhi-Qiang Liu, and Antoni B Chan. 2014. Heterogeneous multi-task learning for human pose estimation with deep convolutional neural network. In Proceedings of the IEEE Conference on Computer Vision and Pattern Recognition Workshops. 482-489.

[Mihoub et al. 2016] Alaeddine Mihoub, Gérard Bailly, Christian Wolf, and Frédéric Elisei. 2016. Graphical models for social behavior modeling in face-to face interaction. Pattern Recognition Letters 74 (2016), 82-89.

[Nguyen et al. 2016] Duc-Canh Nguyen, Gérard Bailly, and Frédéric Elisei. 2016. Conducting neuropsychological tests with a humanoid robot: design and evaluation. In IEEE International Conference on Cognitive Infocommunications (CogInfoCom). 337-342.

[Pan et al. 2011] Sinno Jialin Pan, Ivor W Tsang, James T Kwok, and Qiang Yang. 2011. Domain adaptation via transfer component analysis. IEEE Transactions on Neural Networks 22,2 (2011), 199-210.

[Réveil et al. 2014] Bert Réveil, Kris Demuynck, and Jean-Pierre Martens. 2014. An improved two-stage mixed language model approach for handling out-of-vocabulary words in large vocabulary continuous speech recognition. Computer Speech $\varepsilon$ Language 28, 1 (2014), 141-162.

[Shimodaira 2000] Hidetoshi Shimodaira. 2000. Improving predictive inference under covariate shift by weighting the log-likelihood function. Journal of statistical planning and inference 90, 2 (2000), 227-244.

[Stefanov and Beskow 2016] Kalin Stefanov and Jonas Beskow. 2016. A Multi-party Multi-modal Dataset for Focus of Visual Attention in Human-human and Human-robot Interaction. In Proceedings of the 10th edition of the Language Resources and Evaluation Conference (LREC). ELRA, 4440-4444.

[Sutton et al. 1999] Richard S Sutton, Doina Precup, and Satinder Singh. 1999. Between MDPs and semi-MDPs: A framework for temporal abstraction in reinforcement learning. Artificial intelligence 112, 1 (1999), 181-211.

[Weiss et al. 2016] Karl Weiss, Taghi M Khoshgoftaar, and DingDing Wang. 2016. A survey of transfer learning. Journal of Big Data 3, 1 (2016), 1-40. 